\title{
La política exterior de la dictadura cívico-militar argentina hacia Guatemala $(1976-1983)^{1}$
}

Julieta Rostica ${ }^{2}$

\section{Resumen}

La política exterior de la dictadura cívicomilitar argentina (1976-1983) hacia América Central ha sido comúnmente analizada en función de la participación argentina en la lucha anti y contrarrevolucionaria en $\mathrm{Ni}$ caragua, sin atender a las particularidades históricas de cada uno de los países de la región. Este artículo, en cambio, tiene el objetivo de describir y sistematizar la política exterior de la dictadura argentina hacia Guatemala, identificar cuándo las relaciones entre ambos países se hicieron sistemáticas y, finalmente, ponderar la colaboración argentina en la lucha antisubversiva de dicho país. A partir de la confrontación de fuentes oficiales provistas por diferentes repositorios documentales, afirmamos que las relaciones diplomáticas entre ambos países se hicieron sistemáticas entre 1979 y 1981 y que hubo colaboración en materia de seguridad nacional.

Palabras clave: Política exterior - Dictadura cívico-militar - Coordinaciones represivas - Argentina - Guatemala

\begin{abstract}
The foreign policy of the civil-military dictatorship of Argentina (1976-1983) toward Central America has been commonly analyzed according to Argentina's participation in the anti and counter-revolutionary struggle in Nicaragua, without regard to the historical particularities of each of the countries the region. This article, however, aims to describe and systematize the foreign policy of the dictatorship in Argentina to Guatemala, identify when relations between the two countries became systematic and, finally, weigh the Argentina collaboration in counter-insurgency in that country. From confrontation official sources provided by different document repositories, we affirm that diplomatic relations between the two countries were systematically between 1979 and 1981 and that there was collaboration on national security.
\end{abstract}

Keywords: Foreign policy - Civil-military dictatorship - Repressive coordination - Argentina - Guatemala

\footnotetext{
${ }^{1}$ Trabajo recibido el 14/08/2016. Aceptado el 20/08/2016.

${ }^{2}$ Socióloga (Universidad de Buenos Aires), Magíster en Estudios Latinoamericanos (Universidad Autónoma de México) y Doctora en Ciencias Sociales y Humanas (Universidad Nacional de Quilmes). investigadora del Consejo Nacional de Investigaciones Científicas y Técnicas. Contacto: julietarostica@yahoo.com
} 


\section{Introducción}

Desde el golpe de Estado contrarrevolucionario de 1954, Guatemala comenzó a transitar un proceso de creciente violencia política que alcanzó su punto más álgido con el genocidio perpetrado por el Estado hacia fines de la década del setenta y comienzos de la década del ochenta. El proceso estuvo signado por largas luchas sociopolíticas frustradas en torno a la democratización y por una modernización económica lenta y conservadora, dirigida por una alianza entre elites militares y económicas y partidos políticos de ultraderecha. ${ }^{3}$ Hacia fines de la década de 1970, el movimiento indígena campesino Comité de Unidad Campesina (CUC) coincidió con el nuevo movimiento guerrillero, que desde inicios de la década había cambiado sus estrategias revolucionarias: se había asentado en el altiplano, apostaba a la guerra popular revolucionaria y consideraba a las masas indígenas como una pieza clave de la insurrección social. En 1978, el Ejército Guerrillero de los Pobres también logró articularse con el Consejo Nacional de Unidad Sindical (CNUS), creado en 1976 bajo una visión proletaria y urbana. Las elecciones de julio de 1978 pusieron en evidencia una crisis política que se avecinó con la ruptura de la alianza entre el partido institucional de los militares y el partido tradicional de la extrema derecha. En la ciudad, el CNUS y el Comité de Emergencia de los Trabajadores del Estado, junto a entidades estudiantiles y otras organizaciones sociales fueron las promotoras de las llamadas «Jornadas de octubre de 1978». Hubo paro, barricadas, quema de autobuses y represión por parte de las fuerzas de seguridad del Estado. Estas jornadas desencadenaron huelgas en otras partes de Guatemala, como la de los obreros agrícolas cañeros de la Costa Sur, de enero de 1980, la cual con apoyo del CUC logró adherir a prácticamente cien mil trabajadores. ${ }^{4}$ El gobierno del General Lucas García (1978-1982), especialmente tras la caída de Somoza en Nicaragua en julio de 1979, comenzó a hacer de la represión una práctica masiva que se institucionalizó tras el golpe de Estado de 1982 encabezado por el Gral. José Efraín Ríos Montt y la dictadura institucional de las Fuerzas Armadas que le siguió. ${ }^{5}$ El 2 de enero de 1980, el diario oficial trans-

\footnotetext{
${ }^{3}$ Véase Rouquié, (1994); Torres-Rivas, (2011).

${ }^{4}$ Véase Villagrán Kramer,(1993 y 2004); Grandin, (1997); Torres-Rivas, (2009); Asociación de Investigación y Estudios Sociales (s.f.) y Álvarez Aragón, (2012), (2013a) y (2013b).

${ }^{5}$ Vela, (2014) y Figueroa Ibarra, (2011).
} 
mitió las declaraciones del Ministro de Defensa, Otto Guillermo Spiegler Noriega, quien afirmó la unidad del ejército de Guatemala «como garantía para defender la institucionalidad y la democracia en el país». ${ }^{6}$

En Guatemala, durante este período, más de doscientas mil personas fueron desaparecidas o asesinadas. De las 626 masacres que contabilizó la Comisión para el Esclarecimiento Histórico (CEH), un 95\% se efectuaron entre 1978 y 1984, sobre todo en el área rural, indígena y campesina de Guatemala. En contraste, entre 1962 y 1978 se llevaron a cabo diez masacres en los departamentos caracterizados como ladinos del Oriente del país. ${ }^{7}$ En las masacres se concentraron las ejecuciones arbitrarias (el $69 \%$ del total), las violaciones sexuales (el $41 \%$ del total) y las torturas (el $45 \%$ del total) y en menor escala las desapariciones forzadas. Si bien el trabajo de la CEH afirma que la mayoría de las desapariciones forzadas se llevaron a cabo en los departamentos indígenas, el trabajo de Carlos Figueroa Ibarra, confeccionado a partir de los datos recopilados por el Centro Internacional para Investigaciones en Derechos Humanos y el Grupo de Apoyo Mutuo, señala su concentración en el municipio de Guatemala: el $67 \%$ del período $1970-1979$ y el $47 \%$ del período $1980-1989 .{ }^{8}$ Esto indicaría que fue una práctica especialmente citadina orientada a destruir la organización estudiantil y sindical. Las formas en que se llevaron a cabo las desapariciones forzadas en el ámbito urbano presentan similitudes en relación a la violencia represiva perpetrada por el Estado argentino durante la dictadura cívico-militar de 1976-1983, como puede observarse en los diferentes informes que la Comisión Interamericana de Derechos Humanos hizo sobre Guatemala (1981 y 1983) y en el informe de la comisión

\footnotetext{
${ }^{6}$ Diario de Centro América (2/1/1980).

${ }^{7} \mathrm{CEH},(1999)$.

${ }^{8}$ La CEH (1999) trabajó sobre una muestra de 6159 casos de desaparición forzada y Carlos Figueroa Ibarra (1999) sobre una muestra 4042. De acuerdo al historiador Arturo Taracena Arriola hubo un desbalance en las sedes que ubicó la $\mathrm{CEH}$ : fueron numéricamente mayores en las regiones indígenas que en las regiones ladinas (de población mestiza) lo que provocó un desequilibrio entre la investigación de la violencia en el ámbito urbano y el rural y, por ende, en la recopilación de los datos (2007: 103). En nuestra entrevista a Ana González, quien fue la experta Argentina que estuvo a cargo de dichas regiones ladinas en la investigación de la $\mathrm{CEH}$, esgrimió lo mismo que el historiador. Esto nos mueve entonces a considerar los datos ofrecidos por Carlos Figueroa Ibarra y reflexionar que este tipo de violencia tuvo algunas diferencias con el genocidio perpetrado en las zonas rurales. En otro términos: si bien puede haber colaborado con el genocidio, fue una práctica que tuvo una dispersión cronológica mucho mayor que las masacres y la tierra arrasada, por ejemplo.
} 
de verdad (CEH, 1999). Esta similitud, como otras relacionadas a la organización política de la última dictadura argentina y guatemalteca (19821985), nos condujeron a abordar las relaciones diplomáticas oficiales y no oficiales, legales e ilegales, entre ambos países y a reflexionar sobre una posible hipótesis de trabajo: la colaboración argentina en la lucha antisubversiva en Guatemala. ${ }^{9}$ Esta idea había sido planteada para el caso de Nicaragua, pero apenas esbozada para Guatemala y El Salvador.

El presente artículo tiene el objetivo de describir y sistematizar la política exterior de la dictadura cívico-militar Argentina hacia Guatemala, identificar cuándo las relaciones entre ambos países se hicieron sistemáticas y, finalmente, ponderar la colaboración argentina en la lucha antisubversiva de dicho país. A partir de la confrontación de fuentes oficiales provistas por diferentes repositorios documentales, afirmamos que las relaciones diplomáticas entre ambos países se hicieron sistemáticas entre 1980 y 1981 y que hubo colaboración en materia de seguridad nacional. ${ }^{10}$ Utilizaremos fuentes del Archivo Histórico de la Cancillería Argentina, ${ }^{11}$ de la Biblioteca del Ministerio de Relaciones Exteriores de Argentina, del Archivo y Documentación del Ministerio de Relaciones Exteriores de Guatemala, del Archivo Histórico de la Policía Nacional de Guatemala, del Archivo General del Ejército de Argentina y del Digital National Security Archive de los Estados Unidos.

\section{Mucho ruido y pocas nueces: hacia un estado de la cuestión}

La colaboración de la dictadura cívico-militar argentina a la lucha antisubversiva en Guatemala es planteada en alguna bibliografía académica y periodística, en testimonios y entrevistas, incluso en

\footnotetext{
${ }^{9}$ Rostica, (2013), (2014), (2015) y en prensa.

${ }^{10}$ Se trata de una investigación en curso financiada por el Fondo para la Investigación Científica y Tecnológica (Agencia Nacional de Promoción Científica y Tecnológica, Argentina) basada en documentos secretos pertenecientes al Archivo del Ministerio de Relaciones Exteriores y Culto (MREC): PICT 2013-0751, La colaboración argentina y sus consecuencias en la lucha antisubversiva en Guatemala y El Salvador (1962-1983).

${ }^{11}$ En el presente artículo citamos únicamente los documentos de la Colección Forti pues constituyen los únicos que han sido desclasificados de las cajas existentes sobre Centroamérica. En 2012 hemos iniciado el pedido de desclasificación por el resto de la documentación del Archivo con la que hemos trabajado, expediente 28785/12, pero el trámite sigue en curso.
} 
medios audiovisuales, pero en la mayoría de los casos es una afirmación sustentada en las mismas fuentes.

Las primeras referencias bibliográficas sobre la asistencia argentina en Centroamérica las hizo Eduardo Luis Duhalde en El Estado terrorista argentino. Duhalde decía que en Guatemala «ha sido claramente advertida la presencia intervencionista de los militares argentinos en apoyo de la dictadura de Efraín Ríos Montt, como lo hicieron con su antecesor, general Romeo Lucas García», afirmación que se sostenía por las denuncias del movimiento revolucionario guatemalteco y por las declaraciones de Elías Barahona. Indicaba que operaba, en ese entonces, «una misión militar argentina integrada por oficiales del Ejército y la Armada, especializada en la represión popular mediante la aplicación de la 'técnica argentina'» y que el ex - prisionero Emeterio Toj Medrano, quien había logrado huir en noviembre de 1981 de su cautiverio en el cuartel general Justo Rufino Barrios, corroboraba la presencia argentina en las tareas de interrogación y represión. Su hipótesis se respaldaba, asimismo, por la sesión sobre Guatemala del Tribunal Permanente de los Pueblos, realizada en Madrid del 27 al 31 de enero de 1983.12 Pero esta afirmación, nuevamente, se basaba en el testimonio de Elías Barahona. Éste fue un infiltrado del Ejército Guerrillero de los Pobres en el Ministerio del Interior durante el gobierno de Lucas García. A comienzos de septiembre de 1980, desde Panamá, declaró que los gobiernos de Israel, Chile y Argentina proporcionaban la mayor parte de la ayuda y asesoría militar que el gobierno demandaba y que había unos doscientos oficiales guatemaltecos cursando estudios en las academias argentinas especializándose en técnicas de interrogatorio y tácticas de represión. Más adelante, Barahona declaró que «la política de terror es fomentada y alimentada por los gobiernos de Estados Unidos, Israel, Chile y Argentina, que han intervenido en los asuntos internos de Guatemala abierta y encubiertamente» ante el Tribunal Permanente de los Pueblos de 1983. ${ }^{13}$

Alejandro Dabat y Luis Lorenzano y Oscar Raúl Cardoso, Ricardo Kirschbaum y Eduardo Van Der Kooy en sus trabajos sobre Malvinas ${ }^{14}$

\footnotetext{
${ }^{12}$ Duhalde, (1983): 323-325.

${ }^{13}$ Testimonio, Elías Barahona, Centroamérica, mayo de 1982, Centro Académico de la Memoria de Nuestra América (CAMeNA), Universidad Autónoma de la Ciudad de México, caja 141, expediente T GT3 y caja 9, expediente B GT8, serie Guatemala, sección régimen político y gobierno.

${ }^{14}$ Dabat y Lorenzano, (1984) y Cardoso, Kirschbaum y Van Der Kooy, (1983).
} 
hicieron referencias a la participación Argentina en Centroamérica, pero sin entrar específicamente al caso de Guatemala:

«la Argentina había intentado sin éxito evitar el derrocamiento del dictador nicaragüense Anastacio Somoza proveyéndole hasta el final armas y dinero. Luego envió asesores -expertos en 'contrainsurgencia', especialidad que registró un notorio crecimiento en la Argentina después de 1976- a Honduras y Guatemala, y Galtieri se dispuso incluso a auxiliar a los restos del somocismo en el exilio, empeñados en derrocar al nuevo régimen del Frente Sandinista de Liberación». ${ }^{15}$

Estos autores afirmaron que ese emprendimiento externo fue concebido en 1979 por el Estado Mayor General de Ejército, partiendo de la hipótesis de que la Argentina podría «ocupar los espacios vacíos en la lucha continental contra comunismo» dejados por la administración Carter con su política de derechos humanos. Asimismo, que la intervención de Argentina en Centroamérica bajo el gobierno de Galtieri fue acompasada y apoyada por el gobierno de Reagan hasta los primeros meses de 1982, cuando los episodios en las Islas Malvinas condujeron a la deserción argentina en la región. La operación, que fue conocida como «Plan Charlie», consistía en empujar a los «izquierdistas» salvadoreños hacia Honduras, donde el ejército de ese país terminaría con ellos. ${ }^{16}$

Entre los estudios sobre las relaciones internacionales de Argentina hay que destacar el trabajo de Roberto Russell y Juan Tokatlian. ${ }^{17}$ Ellos distinguieron tres períodos en el desarrollo de las relaciones entre Argentina y América Central, siendo el que fue entre 1981 y junio de 1982 (el último de Videla, la totalidad de Viola y parte de Galtieri) en el que se materializó la defensa de la «civilización occidental» fuera del país, coincidentemente con la llegada de Reagan a la presidencia, la redefinición de la «seguridad nacional» como un proyecto internacional y las disputas entre el Poder Ejecutivo Nacional y algunos sectores de las Fuerzas Armadas. Según este trabajo, con el ascenso de Galtieri, sectores como la Sub-jefatura II del Estado Mayor del ejército, quienes estaban acostumbrados a operar en la clandestinidad, encontraron un terreno fértil para «ofrecer» sus «conocimientos especiales de contrainsurgencia» tanto al ejército salvado-

\footnotetext{
${ }^{15}$ Cardoso, Kirschbaum y Van Der Kooy, (1983): 28.

${ }^{16}$ Cardoso, Kirschbaum y Van Der Kooy,(1983): 53.

${ }^{17}$ Russell y Tokatlian, (1986).
} 
reño como a la contra nicaragüiense. En relación a Guatemala, los autores señalan que «durante finales de 1981 y comienzos de 1982, aumentó la participación de militares en el asesoramiento de contrainsurgencia y práctica de tortura en Guatemala y El Salvador. Todo ello, sumado a la provisión de armamentos.» ${ }^{18}$ La participación del régimen militar en América Central se materializó a través de acciones políticas, diplomáticas, militares y económicas, acciones que los autores sustentaron en fuentes hemerográficas de procedencia estadounidense.

Este trabajo fue recuperado en la monumental obra de Cisneros y Escudé. ${ }^{19}$ Allí se distinguió la intervención argentina en Nicaragua -a través del entrenamiento, armas y financiamiento a los contrarrevolucionarios nicaragüenses en Honduras- de la desplegada en Guatemala y El Salvador, que combinó el mantenimiento del statu quo y la defensa del principio de no intervención con el envío de armas y el asesoramiento en técnicas de lucha antisubversiva. De acuerdo a este trabajo, el involucramiento argentino en la región centroamericana comenzó en 1976 y se extendió, al menos, hasta la crisis de Malvinas desatada en abril de 1982.

En el marco de los estudios históricos sobre la Guerra Fría, Ariel Armony afirmó que los perpetradores de la «guerra sucia» en Argentina trasladaron a fines de los años setenta y comienzos de los ochenta su modelo de represión masiva a América Central. Su investigación tuvo por objetivo mostrar que la Argentina decidió «ocupar el lugar de los Estados Unidos en la lucha hemisférica contra el comunismo» cuando la subversión ya no se percibió como una amenaza seria en el ámbito interno. La Argentina, que «en un principio operó como un actor independiente en América Central», se erigió luego «en un sucedáneo calificado en el programa de política exterior del gobierno de Reagan para esa región». ${ }^{20}$ En este libro, Ariel Armony detalló la asistencia militar que Argentina presuntamente ofreció a Guatemala, El Salvador, Honduras y Nicaragua. Señaló que, precisamente, cuando el gobierno de Cárter canceló la asistencia militar a Guatemala, la Argentina pasó a ocupar su lugar. Incrementó las ventas de material militar, se convirtió en uno de los principales proveedores de instrucción en técnicas de inteligencia avanzada para las unidades de la elite del ejército y participó activamente en los escuadrones de la muerte. Según su pesquisa, la ayuda militar a Guatemala data de 1977 y de un

\footnotetext{
${ }^{18}$ Russell y Tokatlian, (1986): 11.

${ }^{19}$ Cisneros y Escudé, (2000).

${ }^{20}$ Armony, (1999): 69.
} 
convenio secreto firmado entre 1982 y 1983; la instrucción en técnicas de inteligencia avanzadas de la fuerzas de élite guatemalteca G-2 de principios de 1980; el entrenamiento avanzado en inteligencia, incluido el uso de «técnicas de interrogatorio» de 200 oficiales guatemaltecos en Campo de Mayo de un convenio secreto firmado entre autoridades militares en octubre de 1981. También mencionó que el personal militar argentino trabajó con el Ejército Secreto Anticomunista, una de las organizaciones paramilitares guatemaltecas de mayor actividad hacia comienzos de los años ochenta. Las referencias de Armony sobre Guatemala son escasas, no hemos podido localizar los documentos oficiales que menciona y varias de sus afirmaciones se sostienen en la literatura anteriormente citada y en fuentes hemerográficas extranjeras. Es una investigación que si bien es insoslayable, no entró en la comparación analítica de los casos Centroamericanos ni en la significación e importancia de la asistencia argentina para cada experiencia histórica nacional; hizo mayor hincapié nuevamente en la lucha antisandinista dejando abierto un frente de investigación específico en los casos de Guatemala y El Salvador.

Ariel Armony en dicha obra agregó que la celebración del Congreso de la Confederación Anticomunista Latinoamericana (CAL) realizada en septiembre de 1980 en Buenos Aires fue central en los procesos de intercambio entre Argentina y América Central. En general, todas las menciones existentes sobre CAL aparecen ligadas a una dimensión de la colaboración argentina en Centroamérica: la de los asesores argentinos y la existencia del Grupo de Tareas Exterior. Este tema fue abordado desde el campo periodístico. En 2005 hubo algunos avances basados en la desclasificación de documentos del Departamento de Estado estadounidense. ${ }^{21}$ Según éstos, muchos de los militares argentinos que participaron en el Plan Cóndor entre 1975 y 1979 integraron luego la comitiva que siguió entre 1978 y 1984 hacia Centroamérica para entrenar a los llamados «contras». Indican, por ejemplo, que el coronel José Osvaldo Riveiro fue el jefe del Grupo de Tareas Exterior en Honduras, quien reportaba directamente a Carlos Suárez Mason, jefe del Estado Mayor de la dictadura militar y presidente del IV Congreso de CAL. Estas fuentes periodísticas señalan que cuando Reagan asumió la presidencia, las Fuerzas Armadas argentinas decidieron convertirse abiertamente en su fuerza expedicionaria en América Central.

${ }^{21}$ Radio Nikzor, (07/03/2005); Clarin, 24/03/2006); Prensa Libre, (30/01/2008) y O’Donnell, (07/03/2010). 
Algunos indicios clave también fueron revelados por los investigadores del Plan Cóndor. Martorell indicó que Argentina encabezaba el grupo y su proyección hacia el exterior con el objeto de influir en la región y especialmente en Centroamérica. ${ }^{22}$ En Guatemala, dijo Stella Calloni, «los servicios de inteligencia argentinos tenían una sede muy particular y desde allí trabajaban en toda la región, muchas veces bajo el disfraz de agencias periodísticas como fue el caso de una llamada Bairpress. Algunos de sus agentes llegaron a infiltrarse en las filas de refugiados de los distintos países del área para realizar espionaje». ${ }^{23}$ Los aportes de estas investigaciones, junto a las de Patrice McSherry, fueron recuperadas en el libro reciente de Baltasar Garzón. ${ }^{24}$

El video de Héctor Francés, un supuesto ex-integrante del Batallón de Inteligencia 601 del ejército argentino, confeccionado bajo captura de los sandinistas, es central para comprender la inserción argentina en Centroamérica. Francés devela cómo funcionaban los asesores argentinos bajo el paraguas de la «teoría de las fronteras ideológicas», la cual expresa la «necesidad de enviar medios de la inteligencia y del ejército a otros países a los efectos de provocar definiciones que den fronteras ideológicas afines». Según Francés, la interrelación entre los Estados Mayores argentino en Honduras, hondureño y nicarangüense, con base en el Estado Mayor General del Ejército argentino y el Batallón de Inteligencia 601, nació a fines de 1980. Por ese entonces, «comienzan a orquestarse en Guatemala el aglutinamiento de los contrarrevolucionarios, ya con la presencia del militar argentino Villegas y de grupos paramilitares en ese país, grupos paramilitares con estrechas vinculaciones a los grupos fascistas de Mario Sandoval Alarcón o a grupos fascistas de El Salvador...» El video fue citado en el Informe preliminar sobre los desaparecidos en Honduras 1980-1993. ${ }^{25}$

\section{La diplomacia bajo las fronteras ideológicas}

La dictadura cívico-militar argentina nunca se limitó a aplicar los postulados de la doctrina de seguridad nacional al interior del país, puesto que los militares argentinos concibieron la seguridad en términos ideoló-

\footnotetext{
${ }^{22}$ Martorell, (1999): 184-185.

${ }^{23}$ Calloni, (2001): 223.

${ }^{24}$ McSherry, (2009) y Garzón, (2015).

${ }^{25}$ Comisionado Nacional de los Derechos Humanos, (2002).
} 
gicos y hemisféricos. Para uno de los intelectuales de las dictaduras militares de Argentina, Osiris Villegas, el comunismo era una ideología supranacional que había evolucionado del concepto de guerra total al de guerra integral. El afirmaba que el teatro de guerra era uno solo y universal; que América se había constituido «en un 'volcán activo', en donde las fuerzas que promueven la guerra revolucionaria comunista se acrecientan día a día en su resuelto accionar contra las repúblicas latinoamericanas»; y que por eso apremiaba «la necesidad de defender nuestra herencia cultural y los ideales de democracia y libertad» en una estrategia que no debía reducirse al plano nacional, sino que debía estar integrada en un orden regional y mundial. ${ }^{26}$ Las «Bases para la Intervención de las Fuerzas Armadas en el Proceso Nacional» reiteraron «la ubicación internacional en el mundo occidental y cristiano». ${ }^{27}$

La defensa de la «civilización occidental» fuera del país y la definición de la «seguridad nacional» como un proyecto internacional que reconocía fronteras ideológicas y no territoriales, constituyeron parte de las premisas de la Operación Cóndor. ${ }^{28}$ Esos postulados se hicieron radicalmente efectivos durante la administración Carter, al punto de poner en el tapete el tan prestigioso principio de no intervención con la participación argentina en el golpe de Estado boliviano y en la contra nicaragüense. Hay que recordar que en la XIII Conferencia de Ejércitos Americanos (Bogotá, 1979) el Estado Mayor Conjunto de las Fuerzas Armadas argentinas (con el asesoramiento de civiles) formularon la llamada «Doctrina Viola», la cual formalizó el supuesto derecho de los ejércitos latinoamericanos a actuar independiente o colectivamente en todo el país de la región donde se produjeran movimientos revolucionarios por la inacción de Estados Unidos. Bajo este prisma hay que estudiar la diplomacia de la dictadura cívicomilitar argentina hacia Guatemala y viceversa. De hecho, a esta reunión llegaron, de Guatemala, el Viceministro de Defensa Coronel Roberto Salazar Asturias, el Coronel Cáceres Rojas, el Director de Inteligencia Callejas y Callejas y Bolaños Chávez, militares que en lo sucesivo gestionaron ante Argentina la formación en inteligencia. ${ }^{29}$

\footnotetext{
${ }^{26}$ Villegas, (1963): 192-193.

${ }^{27}$ Junta Militar, (1980).

${ }^{28}$ McSherry, (2009) y Revista Taller, (2012).

${ }^{29}$ Cable ordinario, de Tiscornia, EGUAT a latina-informaciones, 2 de noviembre de 1979, MREC, Caja AH/0060, Sección 71-comunicaciones, «Guatemala. vol. 89», cr519.
} 
El gobierno de Guatemala se había posicionado tiempo antes en el frente occidental. El 4 de julio de 1977, para saludar al Cono Sur, condecoró con el gran collar de la Orden de Quetzal a los presidentes de las dictaduras militares de Uruguay, Brasil, Paraguay, Chile y Argentina. ${ }^{30}$ Este gesto fue retribuido en octubre de 1978, cuando estuvo en Argentina el ex Ministro de Relaciones Exteriores de Guatemala, Adolfo Molina Orantes, a quien se le otorgó la condecoración gran cruz de la Orden del Libertador General San Martín. ${ }^{31}$

Desde mediados de la década del sesenta, la embajada argentina pedía informes sobre las actividades políticas en general y sobre las consideradas subversivas en Guatemala. Sin embargo, la comunicación se acrecentó cuando la situación política de Guatemala se tornó compleja con las guerrillas y la coyuntura de Nicaragua, Belice y Jamaica. A juicio de la embajada, en 1978, no se podía analizar la «ola de violencia» en Guatemala sin incluirla en el contexto de lo que ocurría en Nicaragua y El Salvador, ya que dichos países vivían «procesos semejantes derivados de una acción subversiva intensa» ${ }^{32}$. Esta «centroamericanización de la lucha revolucionaria», ${ }^{33}$ que se había producido por efecto de la «reacción en cadena», ${ }^{34}$ se tornó realmente peligrosa después del triunfo sandinista.

Debió ocurrir dicho triunfo y la masacre en la embajada de España, para que el embajador argentino comenzara a enviar cables casi diariamente durante el año 1980, informando a Cancillería sobre los asesinatos, desapariciones, atentados relacionados con asuntos políticos, así como la salida a la luz pública de cualquier organización considerada tanto de extrema derecha como de izquierda. Se trataba de un seguimiento minucioso de la vida política guatemalteca. En 1980, precisamente, el Ministerio de Relaciones Exteriores de Argentina creó un Departamento aparte de

\footnotetext{
${ }^{30}$ Memorias de los trabajos efectuados por el Ministerio de Relaciones Exteriores durante el periodo comprendido del I de julio de 1977 al 30 de junio de 1978. Guatemala: Editorial del Ejército, pp. 260-261.

${ }^{31}$ Decreto 2491 del 20 de octubre de 1978.

${ }^{32}$ Nota reservada, de Tiscornia (Embajador) al Subsecretario de Relaciones Exteriores, Embajada argentina en Guatemala (EGUAT), 24 de julio de 1978, MREC, Colección Forti, Guatemala, nr335.

${ }^{33}$ Nota secreta, de Tiscornia al Subsecretario de Relaciones Exteriores, EGUAT, 26 de junio de 1978, MREC, cForti, Guatemala, nr287. Nota secreta, de Tiscornia al Subsecretario de Relaciones Exteriores, EGUAT, 27 de junio de 1978, MREC, cForti, Guatemala, nr290.

${ }^{34}$ Nota secreta, de Tiscornia al Director General de Política, EGUAT, 1 de febrero de 1979, MREC, cForti, Guatemala, nr41.
} 
América Central y Caribe, separándolo del Departamento América Latina. Las memorias de la cancillería argentina señalan que se iniciaba una «nueva política en el área, apoyada en un programa de contactos directos mediante el envío de misiones especiales de alto nivel presididas por el Subsecretario de Relaciones Exteriores y el Subsecretario de Relaciones Económicas Internacionales y, asimismo, a través del incremento de la asistencia a los países de la zona.» ${ }^{35}$

La masacre en la embajada de España motivó al canciller guatemalteco, Castillo Valdés, a solicitar a Argentina que se hiciera cargo de los intereses de Guatemala en España ante el posible rompimiento de las relaciones diplomáticas entre ambos países. Dicha solicitud se basó en la «similitud ideológica» entre los gobiernos de Guatemala y Argentina y la «identidad de problemas» que ambos tenían. ${ }^{36}$ Lo único que motivó el rechazo de este pedido por parte del gobierno argentino fue la presión que Estados Unidos, los países socialdemócratas europeos y los países del llamado Pacto Andino (Colombia, Venezuela, Ecuador, Perú y Bolivia) ejercían sobre la situación de los derechos humanos en Argentina, la cual «sería o podría ser juzgada como apoyando a un estado que ha violado elementales normas de derecho internacional y podría alentar [la] campaña internacional con Argentina». ${ }^{37}$ No obstante, en la Asamblea General de la OEA que se realizó entre el 19 y el 26 de noviembre de 1980, la Argentina reivindicó la posición del gobierno de Guatemala. ${ }^{38}$

Argentina había buscado acrecentar las relaciones con Guatemala desde 1977, coincidentemente con la llegada de Carter a la presidencia. Estas, sin embargo, avanzaron con el cambio de gobierno en 1978 en Guatemala. Cuando asumió Lucas García, el embajador argentino recibió instrucciones de la cancillería de «sondear las posibilidades de acrecentar todo tipo de intercambios». En el curso de dicho mes, julio de 1978, el embajador se entrevistó de forma privada con el presidente y con varios miembros del gabinete. Pidió una audiencia con el ministro de defensa y el

\footnotetext{
${ }^{35}$ Ministerio de RREE y Culto, Memorias de 1980. Buenos Aires: MREC, 1980, p. 103.

${ }^{36}$ Cable secreto, de Tiscornia, EGUAT a latina-política-occidental-informaciones, 5 de febrero de 1980, MREC, cForti, Guatemala, cr63-66.

${ }^{37}$ Cable secreto, de Tiscornia, EGUAT a latina-política-informaciones, 2 de febrero de 1980, MREC, cForti, Guatemala, cr49-52; cable secreto, de Subiza y Gimenez Melo, Departamento América Latina a EGUAT, 4 de febrero de 1980, MREC, cForti, Guatemala, cel2; nota secreta, de Tiscornia al Jefe del Departamento América Latina, EGUAT, 12 de febrero de 1980, MREC, cForti, Guatemala, nr38.

${ }^{38}$ Ministerio de RREE y Culto, Memorias de 1980. Buenos Aires: MREC, 1980.
} 
Jefe de Estado Mayor «para que las relaciones en el campo militar fueran más allá del otorgamiento de becas por parte de Argentina y se estudiara la posibilidad de concertar una más amplia cooperación». De igual modo con el ministro de finanzas y con el ministro de economía. Según palabras del embajador: «las naciones afectadas de América Latina tenían que tratar de ayudarse y cooperar entre sí para reabastecerse -de ser posible dentro de esa área». ${ }^{39}$

Pese a la enorme trascendencia que tuvieron los sucesos ocurridos en la embajada de España y a la negativa por parte de Argentina de representar los intereses de Guatemala en España, comenzaron a establecerse unas intensas relaciones diplomáticas nunca antes llevadas a cabo entre Argentina y Guatemala. Las memorias de la cancillería argentina indican que en 1980 la relaciones bilaterales «sufrieron un vuelco muy favorable pudiendo considerarse como excelentes en virtud del acercamiento entre ambos países, el intercambio de ideas, la cooperación y el mutuo entendimiento puestos de manifiesto en mayo pasado con la visita que realizó a Guatemala el Subsecretario de Relaciones Exteriores argentino, quien en nombre del Canciller argentino invitó al Ministro de Relaciones Exteriores de Guatemala a visitar la República Argentina, visita que se concretó a fines de agosto de 1980.» ${ }^{40}$

En efecto, entre el 4 y 7 de mayo de 1980, viajó una delegación argentina a Guatemala. El subsecretario de relaciones exteriores argentino Cavandoli se entrevistó con el presidente de Guatemala, con el canciller y con algunos ministros. En la conversación que mantuvo con el canciller Castillo Valdez, el subsecretario analizó el «proceso argentino derivado [de la] necesidad de combatir [la] subversión y [el] terrorismo organizados e insuflados desde el exterior con el fin de poner pie en nuestro país utilizando como plataforma para [el] resto [de] América del Sud» y la evolución «favorable» del país «tanto en el plano político como económico, que permitía exhibir hoy a la Argentina como un país donde reina la tranquilidad y seguridad». El subsecretario agregó que «como consecuencia [de la] derrota [del] terrorismo», el país tenía que enfrentar ataques de países considerados «amigos», señalando que la «experiencia argentina podía servir a título de sugerencias válidas para Guatemala que libra idéntica

\footnotetext{
${ }^{39}$ Nota secreta, de Tiscornia al subsecretario de relaciones exteriores, EGUAT, 20 de julio de1978, MREC, cForti, Guatemala, nr326. Nota secreta, de Tiscornia al subsecretario de relaciones exteriores, EGUAT, 21 de julio de1978, MREC, cForti, Guatemala, nr329. ${ }^{40}$ Ministerio de RREE y Culto, Memorias de 1980. Buenos Aires: MREC, 1980, p. 106.
} 
lucha contra el terrorismo, lo cual puede llevarla a un aislamiento político y económico más acentuado aún que el que se pretende para Argentina» $\mathrm{y}$ que, «en tal sentido, era necesario que aquellos países que están en idéntica situación se apoyaran mutuamente» y le ofreció «la colaboración de la comunidad informativa argentina». Recordemos que las «comunidades informativas» eran un organismo conformado por los servicios de inteligencia argentinos locales encargados de recolectar información de la población mediante inteligencia y la contrainteligencia. ${ }^{41} \mathrm{El}$ canciller Castillo «coincidió plenamente con [los] argumentos expuestos, aclarando [que] Guatemala también libra batalla contra [el] terrorismo y que hasta ahora ha podido dominarlo evitando intervención de EE.UU.» En relación a la entrevista que el subsecretario mantuvo con el presidente Lucas García, expresó que lo invitó al país «en virtud [de la] identidad [de] problemas existentes y [la] necesidad [de] afianzar [la] presencia Argentina en países [de] América Central». Lucas García «destacó la cohesión existente en las Fuerzas Armadas contra el comunismo y la importancia que una gran clase media tiene en esta lucha para Guatemala. En este viaje, el subsecretario de relaciones exteriores argentino llevó proyectos del convenio financiero, comercial y de cooperación técnica. ${ }^{42}$

La confraternización se expresó a través de la condecoración con la gran cruz de la Orden del Quetzal al canciller argentino Carlos Pastor por parte de su colega guatemalteco Rafael Castillo Valdez (19 de agosto de 1980). Entre el 25 y 29 de agosto, el canciller y la comitiva guatemalteca fueron recibidos en Argentina. El canciller guatemalteco pedía firmar, prioritariamente, un convenio sobre cooperación científico-técnica (suscrito el 27 de agosto de 1980), visitar al ministro de defensa y realizar una declaración conjunta. En la misma dejaron constancia que el canciller guatemalteco se había entrevistado con el presidente de la dictadura argentina,

\footnotetext{
${ }^{41}$ El objetivo de la comunidad de inteligencia era compartir la información recolectada entre los distintos integrantes sobre el accionar de las organizaciones políticas, gremiales, estudiantiles, etc. Además, identificar y elaborar listas de personas que consideraban peligrosas, abastecer de datos a las jefaturas correspondientes y al Batallón de Inteligencia 601 y luego accionar a través de grupos de tareas u operativos llevados a cabo por unidades de las Fuerzas Armadas. Programa Verdad y Justicia, (2015).

${ }^{42}$ Cable ordinario, de Tiscornia, EGUAT a surre-latina, 25 de abril de 1980, MREC, cForti, Guatemala, cr299. Cable secreto, de Tiscornia, EGUAT a latina-derechos humanos-subsecretaría de relaciones exteriores-política-organismos-informaciones, 6 de mayo de 1980, MREC, cForti, Guatemala, cr314-319. Sobre los convenios véase el cable ordinario, de Tiscornia, EGUAT a económicos-política-latina, 6 de mayo de 1980, MREC, cForti, Guatemala, cr323.
} 
Jorge Videla, con el ministro de relaciones exteriores y culto y con el ministro de defensa. Asimismo, que analizaron temas de interés común para las dos naciones y que, en virtud de las coincidencias, decidían dejar suscritos, como la firme condena al terrorismo. Acompañaron al canciller guatemalteco el Coronel de Infantería Manuel Antonio Callejas y Callejas y Juan José Urruela Villacorta (diputado del Congreso de la República), entre otros. La presencia de Callejas y Callejas, director de Inteligencia (G2) entre junio de 1980 y marzo de 1982, alertó a los Estados Unidos. ${ }^{43}$ Para ejercer la reciprocidad, el 19 de septiembre de 1980 Eduardo Castillo Valdez fue condecorado por la Argentina (Decreto 1974).

A estos encuentros le siguieron otros. El 10 de noviembre de 1980, el vicepresidente de Guatemala, el Coronel Oscar Mendoza Azurdía y su comitiva viajaron a la Argentina y pidieron una audiencia con el General Videla. ${ }^{44}$ Unos días después, del 26 al 29 de noviembre, una delegación argentina, a pedido insistente de Lucas García, viajó a Guatemala a participar de la III Conferencia de Ministros y Jefes de Planificación de América Latina. ${ }^{45}$

Estos viajes de ida y de vuelta tenían que ver con la negociación de un convenio sobre cooperación científico-técnica (suscrito el 27 de agosto de 1980), de uno financiero por un préstamo de 30 millones de dólares (firmado el 24 de noviembre de 1980 entre el Banco Central argentino y el Banco de Guatemala) ${ }^{46}$ y de uno comercial (firmado el 7 de octubre de 1982).

\footnotetext{
${ }^{43}$ Cable secreto, de Lertora, EGUAT a ceremonial-política-central-gabinete-cultura, 8 de agosto de 1980, MREC, cForti, Guatemala, cr529-535. Cable secreto, de Ros y Freixas, Departamento América Central y Caribe a EGUAT, 22 de agosto de 1980, MREC, cForti, Guatemala, ce195-201. Declaración Conjunta del 27 de agosto de 1980. Declaración Conjunta del 27 de agosto de 1980. Véanse, también, las Memorias de los trabajos efectuados por el Ministerio de Relaciones Exteriores durante el periodo comprendido del I de julio de 1980 al 30 de junio de 1981. Guatemala: Editorial del Ejército, agosto de 1982, pp. 218 y 258. Cable confidencial, de Melvin Sinn, United States Embassy Guatemala, a Department of State, «The Argentine «Connection»: Bits and Pieces», 16 de septiembre de 1980, Digital National Security Archive, Collection Argentina 1975-1980, 005922.

${ }^{44}$ Oficio Nro. 65. de EGUAT a Canciller Brigadier Mayor D. Carlos Washington Pastor, 29 de octubre de 1980, MREC, AH/0042, «Cables 1980».

${ }^{45}$ Ministerio de RREE y Culto, Memorias de 1980. Buenos Aires: MREC, 1980, p. 107. ${ }^{46}$ Cable ordinario, de Lertora, EGUAT a económicos-central, 5 de noviembre de 1980, MREC, cForti, Guatemala, cr740. Véase también Banco Central de la República Argentina, Comunicación B 838, 10 de octubre de 1983.
} 


\section{La diplomacia en seguridad nacional}

La información en materia de seguridad nacional provista por organismos oficiales se cursó cifrada y, en ocasiones, no pasó por la embajada. ${ }^{47} \mathrm{El}$ recuento de reuniones informadas en el apartado previo no fueron las únicas, pues un cable secreto nos informa que oficiales de las Fuerzas Armadas estuvieron en Guatemala y que «una delegación militar guatemalteca e incluso su ministro de defensa viajaron a Buenos Aires», pero que la representación diplomática de Argentina en Guatemala no fue informada. ${ }^{48}$

En efecto, el Ministro de Defensa de Guatemala, Otto Guillermo Spiegler Noriega, durante 1979 viajó al menos en dos oportunidades a la Argentina ${ }^{49}$ y su hijo se graduó en el Colegio Militar de la Nación en $1980 .^{50}$ En enero de 1980 hubo un cambio de gabinete y en sus funciones lo reemplazó el Gral. Aníbal Guevara (quien fungía como Jefe del Estado Mayor General del Ejército). Spiegler Noriega pasó a ser embajador guatemalteco en Argentina. Mientras estuvo a cargo del Ministerio de Defensa, Spiegler Noriega gestionó ante Brasil, Chile, Colombia, China, España, Honduras y México diferentes espacios para que oficiales guatemaltecos pudiesen asistir a diversos cursos en academias militares, pero solo a la Argentina le solicitó becas para la formación en inteligencia. ${ }^{51} \mathrm{Si}$ bien tradicionalmente Argentina ofrecía becas al ejército de Guatemala, recién en

\footnotetext{
${ }^{47}$ Desde julio de 1978 «toda comunicación cablegráfica relacionada con viajes o desplazamientos de [la] comisión militar, miembros de las Fuerzas Armadas o servicio de inteligencia» se cursó cifrada. La información «cifrada» era información escrita con un código (una especie de alfabeto) que cambiaba todas las semanas como mecanismo de seguridad en el intercambio de información secreta. Circular telegráfica, 20 de julio de 1978, MREC, cForti, El Salvador, ctl31.

${ }^{48}$ Nota secreta, de Tiscornia, EGUAT a Subsecretario de Relaciones Económicas Internacionales, 5 de enero de 1979, MREC, cForti, Guatemala, nr7.

${ }^{49}$ Nota secreta, de Tiscornia, EGUAT a Subsecretario de Relaciones Económicas Internacionales, 5 de enero de 1979, MREC, cForti, Guatemala, nr7 y Oficio, Del Cuerpo de Detectives, Policía Nacional, al Coronel de Infantería Germán Chupina Barahona, Guatemala, 30 de marzo de 1979, oficio n ${ }^{\circ}$ 7340, AHPN, Doc 1268609, 2791786_1000. Disponible en: https://ahpn.lib.utexas.edu/es/search/documento/1268609?s= 48434011862007\#page/2/mode/lup.

${ }^{50}$ Ejército Argentino, Boletín Reservado, No 4871, 25 de abril de 1980.

${ }^{51}$ Memorias de los trabajos efectuados por el Ministerio de Relaciones Exteriores durante el periodo comprendido del I de julio de 1979 al 30 de junio de 1980. Guatemala: Editorial del Ejército, mayo de 1981, pp. 230 y 242-243.
} 
1978 abrió el curso de Inteligencia para Oficiales Extranjeros (COE-600). El objetivo del curso era «proporcionar conocimientos técnicos profesionales, especialmente relacionados con la LCS» y estaba dirigido a 14 oficiales subalternos de países invitados y 2 oficiales del ejército argentino. ${ }^{52}$ La sigla LCS aparecía en los documentos de la dictadura significando «lucha contra la subversión». A dicho curso asistieron, en ese año, nada menos que José Mauricio Rodríguez Sánchez y Carlos Enrique Pineda Carranza y en 1979 Francisco Marín Golib y Juan Pablo Soto Jiménez. ${ }^{53}$ Rodríguez Sánchez fue quien siguió a Callejas y Callejas en la dirección de la Sección de Inteligencia G-2 (abril de 1982 y abril de 1983), juzgado en 2013 junto a José Efraín Ríos Montt por los delitos de genocidio y deberes contra la humanidad. Pineda Carranza fue jefe de negociado en el Estado Mayor General del Ejército (1978-diciembre de 1980) y un oficial de la G-2 desde 1980.

En junio de 1980, el Viceministro de Defensa Coronel Salazar Asturias destacó, en una conversación con el agregado militar de Argentina, la «necesidad de ampliar [la] cooperación argentina especialmente en materia de 'cursos de inteligencia'» ${ }^{54}$ y pocos meses después, Callejas y Callejas estuvo en el país. Según algunos documentos estadounidenses, ninguno de los convenios que hemos mencionado en el apartado anterior contemplaba la asistencia militar. En éstos, sin embargo, se sugiere que ésta podría haberse formalizado por separado y que Callejas y Callejas había informado sobre la formación militar de civiles del Ministerio de Defensa de Guatemala en Argentina. ${ }^{55}$ Inmediatamente las plazas para los guatemaltecos aumentaron. Ese año llegó Juan Adenolfo Gálvez del Cid, Leonel De León Guillén, Horacio Soto Salam, Carlos Eddie Radford Bonilla, José Manuel Rivas Ríos y Byron Humberto Barrientos Día, quien en la

\footnotetext{
${ }^{52}$ Ejército Argentino, Boletín Reservado, No 4738, 21/10/1977.

${ }^{53}$ Ejército Argentino, Boletín Reservado, No 4764, 07/04/1978; Ejército Argentino, Boletín Reservado, No 4820, 04/05/1979 y Ejército Argentino, Boletín Reservado, № 4837, 31/08/1979.

${ }^{54}$ Cable secreto, de Tiscornia, EGUAT a América Central-política-informaciones, 2 de junio de 1980, cr389-394.

${ }^{55}$ Cable confidencial, de Melvin Sinn, United States Embassy Guatemala, a Department of State, «Growing Argentine Involvement in Guatemala», 13 de agosto de 1980, Digital National Security Archive, Collection Argentina 1975-1980, 005199 y Cable confidencial, de Melvin Sinn, United States Embassy Guatemala, a Department of State, «The Argentine «Connection»: Bits and Pieces», 16 de septiembre de 1980, Digital National Security Archive, Collection Argentina 1975-1980, 005922.
} 
actualidad se encuentra sindicado por torturas y desapariciones forzadas en la zona de Cobán mientras trabajaba para la misma Dirección de Inteligencia del ejército. ${ }^{56}$ En 1981 tomaron el curso Mario Mérida, quien en los noventa estuvo a cargo de la Dirección de Inteligencia y Alfonso Tarzo De León Maldonado; ${ }^{57}$ y en 1982 Fernando Alberto Morales Sandoval y Julio Alpírez, jefe de los kaibiles en los años noventa. ${ }^{58}$ Para contextualizar el peso de la representación guatemalteca en los COE-600 es importante mencionar que Guatemala, España y Ecuador encabezaron la lista: 22 oficiales de cada país recibieron la Aptitud Especial de Inteligencia. ${ }^{59}$

La importancia que puede haber tenido la formación en inteligencia ofrecida por el gobierno argentino es difícil de evaluar. En términos generales puede decirse que era un campo en el que los militares argentinos se sentían con conocimientos y experiencia. De hecho, hubo instructores argentinos de inteligencia en la Escuela de las Américas, en la zona del Canal de Panamá. En el caso especial de Guatemala, las entrevistas de Schirmer pueden servir para ponderar la importancia del curso argentino. Un ex funcionario administrativo de la G-2 le relató que:

«la inteligencia que aprendimos de los Estados Unidos era muy buena en el campo, en la guerra vinculada, pero no era eficiente en el campo de la guerra a la guerrilla. Entonces, cuando Guatemala fue aislada entre 1978 y 1984, recurrimos a países como Argentina y Uruguay que habían tenido problemas grandes (...) Entonces, desarrollamos nuestra propia inteligencia antisubversiva con el apoyo de esos países. Nosotros vimos un viraje total a los conceptos de manejo de inteligencia de guerra». ${ }^{60}$ (Díaz López, entrevista)

En esa misma obra, Gramajo le afirmó a la investigadora que «quien nos entrenó mucho en inteligencia fue Argentina.» Según su relato, se controlaba el uso del agua y la electricidad en las casas de sospechosos en la ciudad de Guatemala. Un sistema computarizado israelí se combinaba con una sofisticada red computarizada de análisis que se había desarrollado en Argentina. Con esta tecnología se movilizaba a las tropas del ejército para

\footnotetext{
${ }^{56}$ Ejército Argentino, Boletín Reservado, No 4871, 25 de abril de 1980 y Ejército Argentino, Boletín Reservado, $N^{\circ} 4893,26 / 09 / 1980$.

${ }^{57}$ Ejército Argentino, Boletín Reservado, No 4934, 04/05/1981.

${ }^{58}$ Ejército Argentino, Boletín Reservado, No 4996, 26/04/1982.

${ }^{59}$ Dana, (2004): 393.

${ }^{60}$ Schirmer, (1999): 265.
} 
rodear zonas enteras y registrar casas en diferentes zonas de la ciudad. Así, según una entrevista a un coronel de la G-2, a mediados de 1981 se hizo una redada en alrededor de treinta escondites y depósitos de armas de la guerrilla (sobre todo de la ORPA) en la ciudad de Guatemala que logró destruir una extensa red urbana. ${ }^{61}$

Según Jennifer Schirmer, durante el gobierno de Lucas García la represión política se ideó en el Centro de Reunión de Información y Operaciones (CRIO), un grupo integrado por el Ejecutivo y los jefes de los cuerpos de seguridad: el coronel Montalván Batres quien estaba a cargo de la oficina Especial de Apoyo Presidencial y Servicios de Comunicación, Donaldo Álvarez Ruiz, Ministro de Gobernación y el coronel de la G-2 Germán Chupina, quien dirigía la Policía Nacional. En la oficina presidencial se controlaban las actividades de información en el país y las operaciones de inteligencia en la ciudad. En la ciudad de Guatemala, la policía, el ejército y los escuadrones de la muerte fueron los responsables de los asesinatos políticos. ${ }^{62}$

En Argentina, si bien la llamada «lucha contra subversión» se desplegó sobre todo en el ámbito urbano, la policía quedó formalmente subordinada el ejército. De todos modos, la experiencia resultaba interesante para Donaldo Álvarez Ruiz, el principal responsable de la masacre en la embajada de España, quien visitó el país de forma privada el 19 de noviembre de 1979. El mismo arribaba al país para interiorizarse sobre la organización de la Policía Federal Argentina. ${ }^{63}$ Argentina ofreció numerosas becas a Guatemala para los cursos que impartía la Policía Federal. De acuerdo al Plan Anual de Becas para Policías Latinoamericanos de la Policía Federal Argentina, en 1980 las becas eran muchas y en la mayoría de los cursos se contemplaba la formación en inteligencia. ${ }^{64}$

El año 1980 fue clave, pues en febrero se creó la agregación militar en Guatemala, la cual tuvo jurisdicción también sobre Honduras, El Sal-

\footnotetext{
${ }^{61}$ Schirmer, (1999): 267.

${ }^{62}$ Schirmer (1999): 263-264.

${ }^{63}$ Cable ordinario, de Tiscornia, EGUAT a latina-política-informaciones, 16 de noviembre de 1979, MREC, Caja AH/0060, Sección 71-comunicaciones, «Guatemala. vol. 89», cr551. Cable secreto, de Tiscornia, EGUAT a latina-política-informaciones, 10 de noviembre de 1979, MREC, Caja AH/0060, Sección 71-comunicaciones, «Guatemala. vol. 89», cr539-41.

${ }^{64}$ Plan anual de becas año 1980 para Policías Latinoamericanos (18 páginas), AHPN, Doc 1377393. Disponible en https://ahpn.lib.utexas.edu/es/search/documento/ 1377393 ? $=1377393$ \#page/13/mode/lup.
} 
vador, República Dominicana y Haití. El cargo lo ejerció el coronel Juan Arturo Ehlert desde marzo de $1980^{65}$ y desde 1981 el coronel Humberto Pompilio Ferrucci. En 1980 las más altas autoridades de la Zona Militar «General Aguilar Mariscal Zavala», la zona militar de la capital guatemalteca, estuvieron en Argentina. El 22 de noviembre de 1980, una misión militar guatemalteca llegó al país, presidida por el Jefe del Estado Mayor del Ejército Luis René Mendoza Palomo e integrada por el General de Brigada Héctor López Fuente (Comandante de la Zona Militar «General Aguilar Mariscal Zavala»), el Coronel Oscar Cuyun Medina (Comandante de la Brigada Mariscal Zavala) y el Capitán Rudy Flores Molina. Mendoza Palomo había expresado que dicha visita sería muy positiva «ya que es necesario que ambos países estén unidos y que exista entre ellos una efectiva cooperación.» ${ }^{66}$ En 1981 la Argentina condecoró a esos militares con la Orden de Mayo al Mérito Militar mediante el Decreto 55. ${ }^{67}$ Pocos meses después, una misión militar argentina llegó a Guatemala. Viajaron el Jefe del Estado Mayor del Ejército el General José Vaquero, el General Héctor N. Iglesias, el Coronel Pedro Corabella y el Mayor José C. Hilgert. ${ }^{68}$ Sobre esta última existe evidencia en el Archivo Histórico de la Policía Nacional de Guatemala (AHPN). Allí se consigna el ingreso al país de los funcionarios argentinos, la visita de Vaquero junto a López Fuentes a Antigua Guatemala, quien fue Jefe del Estado Mayor del Ejército durante la dictadura encabezada por Ríos Montt, y, curiosamente, la salida de la delegación militar argentina hacia Miami y luego hacia Argentina. ${ }^{69}$ Esa

${ }^{65}$ Cable secreto, de Subiza, América Latina a EGUAT, 19 de febrero de 1980, MREC, cForti, Guatemala, ce30. Cable secreto, de Sonschein y Jorgensen, Ceremonial a EGUAT, 22 de abril de 1980, MREC, cForti, Guatemala, ce75. Cable ordinario, de Balbi, América Central a EGUAT, 12 de junio de 1980, MREC, cForti, Guatemala, cel34.

${ }^{66}$ Cable secreto, de Tiscornia, EGUAT a central-política-informaciones-ceremonial, 23 de noviembre de 1980, MREC, cForti, Guatemala, cr798.

${ }^{67}$ En 1977 la Gran Cruz de la Orden de Mayo al Mérito fue otorgada a los guatemaltecos Alfredo Obiols Gómez y a Jaime Martínez y la del Gran Oficial a Angel Anibal Guevara Rodríguez.

${ }^{68}$ Cable secreto, de Claraso de la Vega, Departamento América Central y Caribe a EGUAT, 16 de marzo de 1981, MREC, cForti, Guatemala, ce88. Cable secreto, De Simone, Subsecretaría general a EGUAT, 16 de marzo de 1981, MREC, cForti, Guatemala, ce89.

${ }^{69}$ Informe, del Jefe del Cuerpo de Radio Patrullas de la Policía Nacional al Coronel Germán Chupina Barahona, Director General de la Policía Nacional, 6 de abril de 1981, AHPN, GT PN 31-02 S008, F58118, Nº de documento PN 1737/Ioc; Informe, del Jefe del Cuerpo de Radio Patrullas de la Policía Nacional al Coronel Germán Chupina Barahona, Director General de la Policía Nacional, 8 de abril de 1981, AHPN, GT PN 31-02 S008, F58115, No de documento PN 1772/hcb; Informe, del Jefe del Cuerpo de Radio 
visita seguramente está relacionada con otra que finalizó el 30 de abril de 1981 que no consta en las memorias oficiales de ninguna cancillería. En el AHPN se constata la salida de Guatemala de una delegación militar argentina y de una estadounidense en un mismo avión de la Fuerza Aérea Argentina. ${ }^{70}$

Los intercambios oficiales y no oficiales se trasladaron a los manuales. El Manual de Guerra Contrasubversiva (MGC) del Ejército de Guatemala de comienzos de los años ochenta consignó que para su elaboración se consideraron las experiencias de Argentina, Francia y Perú. Es un corpus muy interesante, por cuanto define al enemigo interno por la llamada «'Guerra Revolucionaria' y la subversión en el país», un lenguaje que no había sido de uso corriente en Guatemala con anterioridad. El MGC, en una breve bibliografía cita, precisamente, el libro de Osiris Villegas, Guerra revolucionaria comunista, y un manual del ejército argentino del año 1962: Instrucciones para la lucha contra la subversión. Seguramente se trataba del reglamento Instrucción de lucha contra elementos subversivos de 1976.

\section{Breves reflexiones finales}

En este artículo hemos procurado describir sucintamente y sistematizar la política exterior de la dictadura cívico-militar argentina hacia Guatemala, con el objetivo de mostrar su especificidad en relación a los otros países de la región. Hemos intentado hacer esta tarea utilizando las fuentes provistas por una serie de repositorios documentales oficiales no utilizados por otras investigaciones precedentes. El trabajo indica que las relaciones diplomáticas entre ambos países se hicieron sistemáticas entre 1979 y 1981 y que hubo colaboración en materia de seguridad nacional.

Las relaciones diplomáticas oficiales fueron asiduas y la cooperación en diversos ámbitos se logró formalizar en diferentes convenios. La cooperación en seguridad nacional se efectuó de forma oficial y secreta. No hay dudas de que Argentina ofreció becas para la formación militar de

Patrullas de la Policía Nacional al Coronel Germán Chupina Barahona, Director General de la Policía Nacional, 10 de abril de 1981, AHPN, GT PN 31-02 S008, F58114, Nº de documento PN 1805/hcb.

${ }^{70}$ Informe, del 2 do. Jefe del Cuerpo de Radio Patrullas de la Policía Nacional al Coronel Germán Chupina Barahona, Director General de la Policía Nacional, 30 de abril de 1981, AHPN, GT PN 31-02 S008, F58117, No de documento PN 2121/hcb. 
guatemaltecos, que impartió cursos de inteligencia y lucha antisubversiva para oficiales de Guatemala, así como becas para la formación de cuerpos policiales, los cuales también impartían cursos de inteligencia. Todos estos intercambios se recuperaron y expresaron en el Manual de Guerra Contrasubversiva de Guatemala.

A diferencia de los otros países de la región, la participación Argentina en la lucha antisubversiva que libraba el gobierno de Guatemala tuvo que ver más con la formación de cuadros en inteligencia de quienes serían luego jefes de la Dirección de Inteligencia, que con la venta de armas (que no dejaron de ofrecer) o el envío de asesores. Estos últimos llegaron a la Escuela de Comando y Estado Mayor de Honduras y lograron cambiar el «programa original de origen norteamericano según [el] modelo [de la] escuela [de] EE. UU. en Panamá, y establecieron [un] plan de estudios y ejercicios sobre [la] base [de] necesidades propias, buscando formar doctrina nacional genuina de defensa, con procedimientos similares a [los] utilizados en nuestro país. $\gg^{71}$ Posiblemente a través de Honduras, las instituciones represivas guatemaltecas recibieron colaboración Argentina. Un posible enlace puede haber sido Cuyun Medina, quien pasó a ser embajador guatemalteco en Honduras. Pero esa línea de indagación excede los objetivos del presente artículo.

Creemos que la relaciones entre ambos gobiernos se hicieron sistemáticas entre 1979 y 1981 por tres razones concatenadas: por la adhesión del gobierno argentino a los principios de la guerra ideológica; por la interpretación que el gobierno argentino hizo del triunfo de la revolución sandinista (julio 1979) y de las consecuencias que podía generar en la región; $\mathrm{y}$, finalmente, por el posicionamiento del gobierno argentino y guatemalteco en relación a la política del gobierno de Jimmy Carter. Entre 1978 y 1980 Guatemala estuvo fuera del programa de ventas militares, de entrenamiento y ventas comerciales de Estados Unidos por su alto índice en la violación a los derechos humanos y Argentina se encontraba en una situación prácticamente idéntica.

${ }^{71}$ Cable cifrado, de Ossorio Arana, Honduras, 18 de agosto de 1982, MREC, cForti, Honduras, cr296. 


\section{Bibliografía}

ÁlVAREZ ARAGÓN, Virgilio, et al, (2012, 2013a y 2013b), Guatemala: historia reciente (1954-1996), Tomos: Proceso político y antagonismo social; La dimensión revolucionaria; Pueblo indígenas, actores políticos. Guatemala: FLACSO.

ARMONY, Ariel, (1999), La Argentina, los estados unidos, y la cruzada anticomunista en América Central (1977 - 1984). Buenos Aires: Universidad de Quilmes.

CALlONI, Stella, (2001), Operación Cóndor. Pacto Militar. México: La Jornada.

CARDOSO, Oscar Raúl; KIRSCHBAUM, Ricardo y VAN DER KOOY, Eduardo, (1983), Malvinas. La trama secreta. Buenos Aires: Sudamericana.

CISNEROS, Andrés y ESCUDÉ, Carlos, (2000), Historia general de las relaciones exteriores de la República Argentina, Tomo XIV. Buenos Aires: Grupo Editor Latinoamericano.

DABAT, Alejandro y LORENZANO, Luis, (1984), The Malvinas and the end of militar rule. Londres: Verso.

DANA, Horacio Ángel, (2004), Historia de la Escuela de Inteligencia. Buenos Aires.

DUHALDE, Eduardo Luis, (1983), El Estado terrorista Argentino. Buenos Aires: El Caballito.

FIGUEROA IBARRA, Carlos, (1999), Los que siempre estarán en ninguna parte (La desaparición forzada en Guatemala). México: Universidad de Puebla.

FIGUEROA IBARRA, Carlos, (2011), El recurso del miedo. Estado y terror en Guatemala. Guatemala: F\&G editores.

GARZÓN, Baltasar, (2015), Operación Cóndor: 40 años después. Buenos Aires: Infojus.

GRANDIN, Greg, (1997), «To end with all these evils», Latin American Perspectives, vol. 24, nro. 2: 7-34.

MARTORELL, Francisco, (1999), Operación Cóndor. Santiago de Chile: LOM.

MCSHERRY, J. Patrice, (2009), Los Estados depredadores: La Operación Cóndor y la guerra encubierta en América Latina. Santiago de Chile: LOM. 
VILLEGAS, Osiris, (1963), Guerra Revolucionaria Comunista. Buenos Aires: Pleamar.

Programa Verdad y Justicia, (2015), El Batallón de Inteligencia 601. Buenos Aires: Infojus.

ROSTICA, Julieta, (en prensa) «La última dictadura guatemalteca en perspectiva comparada (1982-1985)», en Roberto García Ferreira y Arturo Taracena Arriola compiladores, Guerra Fría y anticomunismo en Centroamérica. Guatemala: FLACSO Guatemala.

ROSTICA, Julieta, (2013), «Una agenda de investigación pendiente: la política exterior de la dictadura militar argentina hacia Guatemala (1976-1983)», Boletín (AFEHC), $\mathrm{N}^{\circ}$ 59, Toulouse. Disponible en http://www.afehc-historia-centroamericana.org/ ?action $=$ fi_aff\&id $=3661$.

ROSTICA, Julieta, Las legitimaciones de la dictadura militar de Guatemala. 1982-1985, Revista Aletheia, Vol. 4, No 8, Facultad de Humanidades y Ciencias de la Educación, Universidad Nacional de La Plata, 2014. Disponible en http://www.aletheia.fahce.unlp.edu.ar/ numeros/numero-8/dossier/las-legitimaciones-de-la-dictadura-militar-de-guatemala-1982-1985

ROSTICA, Julieta, (2015), «Las dictaduras militares en Guatemala (19821985) y Argentina (1976-1983) en la lucha contra la subversión, Latinoamérica». Revista de Estudios Latinoamericanos, $\mathrm{N}^{\circ}$ 60, Centro Coordinador y Difusor de Estudios Latinoamericanos, Universidad Nacional Autónoma de México. pp. 13-52. Disponible en http: //www.cialc.unam.mx/web_latino_final/archivo_pdf / Lat60-13.pdf

ROUQUIÉ, Alain, (1994), Guerras y paz en América Central. México: Fondo de Cultura Económica.

RUSSELL, Roberto y TOKATLIAN, Juan, (1986), Argentina y la crisis centroamericana, 1976-1985. Buenos Aires: FLACSO.

SCHIRMER, Jennifer, (1999), Las intimidades del proyecto politico de los militares en Guatemala. Guatemala: FLACSO.

TARACENA ARRIOLA, Arturo, (2007), «Historia, memoria, olvido, conflicto armado y violaciones de los derechos humanos. Los avatares de la Comisión de Esclarecimiento Histórico de Guatemala», en María Rosaria Stabili (coord.), Entre historias y memorias: los desafíos metodológicos del legado reciente de América Latina. Madrid: Iberoamericana, pp. 91-112. 
TORRES-RIVAS, Edelberto, (2009), «Ocho claves para comprender la crisis en Centroamérica», en Edelberto Torres-Rivas, Centroamérica. Entre revoluciones y democracia. Buenos Aires: CLACSO y Prometeo, pp. 107-141.

TORRES-RIVAS, Edelberto, (2011), Revoluciones sin cambios revolucionarios. Guatemala: F\&G editores.

VELA, Manolo, (2014), Los pelotones de la muerte. México: El Colegio de México.

VILLAGRÁN KRAMER, Francisco, (1993), Biografía politica de Guatemala. Los pactos politicos de 1944-1970. Guatemala-Costa Rica: FLACSO.

VILLAGRÁN KRAMER, Francisco, (2004), Biografía politica de Guatemala. Años de Guerra y años de Paz. Guatemala: FLACSO. 